\title{
Impact Environnemental de L'adoption des Variétés Ameliorées de Soja dans le Département du Borgou au Nord du Bénin
}

\author{
Oloumiladé Obafémi Marcellin, \\ Akpo Ibidon Firmin, \\ Yabi Afouda Jacob,
}

Laboratoire d'Analyse et de Recherches sur les Dynamiques Économiques et Sociales (LARDES), Université de Parakou, Bénin

Doi:10.19044/esj.2019.v15n28p148 URL:http://dx.doi.org/10.19044/esj.2019.v15n28p148

Résumé

Les variétés améliorées ont été introduites dans l'agriculture dans l'objectif d'accroitre les rendements. Mais leur production suscite beaucoup de controverses en raison des préoccupations d'ordre environnemental. Elles sont, en effet, associée à des produits agrobiochimiques tels que les engrais, herbicides, insecticides etc. Si l'efficacité de ces produits a été prouvée, leur manipulation pose de sérieux problèmes environnementaux et sanitaires. L'objectif de cette étude est de déterminer l'impact environnemental de l'adoption des variétés améliorées de soja dans le département du Borgou au Bénin. Pour y parvenir, une enquête a été menée dans la zone d'étude en mars 2018 sur 219 producteurs choisis au hasard. Les données obtenues ont été traité à l'aide de l'outil économétrique. Ainsi, l'estimation d'un modèle de Heckman à deux étapes d'une part et d'un modèle de régression linéaire d'autre part ont permis de montrer que l'adoption des variétés améliorées de soja n'affecte nullement la qualité de l'environnement dans la zone d'étude.

Mots clés : Adoption, Variétés améliorées, Heckman, Borgou 


\title{
Environmental Impact of the Adoption of Improved Varieties of Soybeans in the Departement of Borgou in Northern Benin
}

\author{
Oloumiladé Obafémi Marcellin, \\ Akpo Ibidon Firmin, \\ Yabi Afouda Jacob,
}

Laboratoire d'Analyse et de Recherches sur les Dynamiques Économiques et Sociales (LARDES), Université de Parakou, Bénin

\begin{abstract}
Improved varieties have been introduced in agriculture with the aim of increasing yields. But their production is very controversial because of environmental concerns. They are, in fact, associated with agrobiochemical products such as fertilizers, herbicides, insecticides etc. If the effectiveness of these products has been proven, handling them poses serious environmental and health problems. The objective of this study is to determine the environmental impact of the adoption of improved varieties of soybeans in the Borgou department of Benin. To achieve this, a survey was conducted in the study area in March 2018 on 219 randomly selected producers. The data obtained was processed using the econometric tool. Thus, the estimation of a two-stage Heckman model and a linear regression model have shown that the adoption of improved soybean varieties does not affect the quality of the environment in the study area.
\end{abstract}

Keywords: Adoption, Variétés améliorées, Heckman, Borgou

\section{Introduction}

La plupart des pays de l'Afrique au Sud du Sahara ont une économie essentiellement basée sur l'agriculture. Le Bénin n'échappe pas à cette réalité. En effet, le secteur agricole béninois occupe environ $70 \%$ de la population active. Il contribue à hauteur de $23 \%$ à la formation du Produit Intérieur Brut (PIB). De même, il fournit environ $75 \%$ des recettes d'exportation et $15 \%$ des recettes de l'État (Akomagni \& Ichola, 2017). Mais le secteur est peu diversifié et la production alimentaire peu développée.

Pour relever ces différents défis, le Bénin a priorisé dans son Plan Stratégique de Développement du Secteur Agricole (PSDSA) arrimé au 
Programme d'Actions du Gouvernement (PAG), plusieurs filières émergentes dont le soja. En effet, le soja est un produit en pleine expansion dans le pays. Sa production a connu une nette augmentation passant de 12433 tonnes en 2007 à 156901 tonnes en 2016, soit une augmentation de 1 161,97\% en 9 ans (Konnon \& Ahoueya, 2017) ce qui correspond à une variation annuelle de $32,54 \%$ environ. Il représente une plante à croissance rapide et à forte valeur nutritive de par sa teneur en protéines et en énergie, jouant ainsi un rôle important dans l'approvisionnement alimentaire mondial (Batamoussi et al., 2016). Il possède une capacité d'adaptation à des conditions environnementales très variées. Par l'activation de la vie microbienne, la plante de soja améliore la structure du sol (Chogou et al., 2018). Elle fertilise et enrichit le sol puis le protège contre l'érosion (Batamoussi et al., 2016).

Mais la production du soja au Bénin est soumise à un certain nombre de contraintes : faibles niveaux de rendement, déhiscence des gousses à maturité, non disponibilité d'engrais spécifiques, faible compétitivité sur le marché international, faible vulgarisation des techniques améliorées de production, perturbation des aléas climatiques etc.

Cependant, avec l'existence des variétés améliorées à haut rendement et non déhiscentes, la production du soja a acquis des marges de progrès très importantes (Chogou et al., 2018; Njonjo Assani, 2014). En effet, le rendement moyen de ces variétés estimé à $3 \mathrm{t} / \mathrm{ha}$ est nettement supérieur à celui des variétés conventionnelles $(840 \mathrm{~kg} / \mathrm{ha}$ à $1020 \mathrm{~kg} / \mathrm{ha})$. Elles ont été introduites au Bénin grâce à plusieurs institutions et Organisations NonGouvernementales et particulièrement l'Institut International d'Agriculture Tropicale (IITA) qui s'est investie dans l'amélioration variétale du soja pour proposer aux populations les lignées TGx (Tropical Glycine Cross) qui constituent des lignées africaines de soja.

Mais la production des variétés améliorées suscite beaucoup de controverses en raison des préoccupations d'ordre environnemental. Elle est, en effet, associée à des produits agrobiochimiques tels que les engrais, herbicides, insecticides etc. (Célestin, 2012). Selon l'auteur, l'avenir de l'agriculture repose désormais sur ces adaptateurs et ces régulateurs agrobiochimiques. Si l'efficacité de ces produits a été prouvée, leur manipulation pose de sérieux problèmes environnementaux et sanitaires (Kanda et al., 2013 ; Adechian et al., 2015). Cette recherche a pour objectif de déterminer l'impact de l'adoption des variétés améliorées de soja sur l'environnement dans le département du Borgou au Bénin.

\section{Méthodologie}

\section{Zone d'étude, échantillonnage et données}

Les données utilisées dans le cadre de ce travail ont étés collectées dans les communes de Pèrèrè, Nikki, Bembéréké et N'Dali (département du 
Borgou). Le choix de ce département comme zone d'étude se justifie par le fait qu'il est le premier producteur de soja au Bénin.

Tableau 1 : Caractéristiques de la zone d'étude

\begin{tabular}{|c|c|}
\hline Variables & Valeurs \\
\hline Pluviométrie & 1200 à $1300 \mathrm{~mm}$ \\
\hline Végétation & $\begin{array}{c}\text { Savane herbeuse, savane arborée et } \\
\text { arbustive, savane boisée et forêt galerie }\end{array}$ \\
\hline Taux de croissance de la population & $4,68 \%$ \\
\hline $\begin{array}{c}\text { Place du département dans la production du } \\
\text { soja }\end{array}$ & $1^{\text {ère }}$ place \\
\hline Densité & 47 habitants au $\mathrm{km}^{2}$ \\
\hline Pourcentage d'agriculteurs dans la population & $67,92 \%$ \\
\hline
\end{tabular}

Source : Cahier des villages et quartiers de ville du département du Borgou, 2016.

Au total, 44 villages ont été parcourus et 219 producteurs ont été enquêtés. Dans chacune des communes, les villages sont choisis par randomisation. Quant au choix des chefs d'exploitations enquêtés à l'intérieur de chaque village, il est fait avec la méthode d'échantillonnage aléatoire et périodique. Le principe est le suivant : dans un village, il est établi une liste ordonnée des producteurs (et productrices) dont le soja constitue la culture principale avec l'aide des responsables des Unions Villageoises des Producteurs de Soja (UVPS). Supposons que le nombre de producteurs remplissant les conditions dans le village est $N$ et $n$ le nombre à enquêter. On détermine la période $f=N / n$. On choisit au hasard un numéro parmi les $N$ producteurs. Ce numéro sera retenu comme celui du premier individu à enquêter dans ce village. Les autres individus sont choisis par bonds successifs croissants ou décroissants de $f$ en $f$ jusqu'à l'obtention des $n$ numéros à enquêter dans le village.

Les données recueillies portent sur les caractéristiques socioéconomiques des enquêtés (sexe, quantité produite en soja, niveau d'éducation, statut d'alphabétisation, âge, superficie totale de terre, taille du ménage, usage d'engrais chimiques et de pesticides etc.) ; les questions relatives au cadre social (appartenance à un groupement ou association de producteurs, contacts avec des agents vulgarisateurs, participation aux expérimentations sur la sélection variétale etc.) et sur les caractéristiques propres aux variétés de soja (déhiscence, rendements etc.).

\section{Rôle théorique de l'innovation dans la détérioration de la qualité de l'environnement}

Selon Adam Smith (1723-1790), la croissance économique résulte de l'accumulation de richesses obtenues à l'aide d'une meilleure organisation des intérêts individuels (Thibault, 1970). Mais d'après la théorie du trade-offs, les bénéfices induits par la modification d'un train de vie peuvent être négativement corrélées à un autre. On s'aperçoit donc que l'analyse de Smith 
souffre d'une réalité : l'évidence des rapports de l'Homme à l'environnement. Waaub (1991) explique que ces rapports sont dominés par la conception selon laquelle l'Homme (créature supérieure) doit disposer d'éléments de la nature (qui lui sont soumis) pour satisfaire ses besoins. Cette idée est en effet à l'origine de l'expansion de la civilisation européenne qui s'est réalisée au $\mathrm{XVI}^{\mathrm{e}}$ siècle sans aucune attention au facteur environnemental. Mais à partir des années 1970, l'inquiétude quant à la capacité de la biosphère à fournir les ressources nécessaires pouvant permettre de poursuivre la croissance économique s'est fait grandissante. Les pays développés s'étaient alors persuadés que la dégradation environnementale l'emportait sur les gains tirés des progrès économique et technologique et qu'elle affectait peut-être de manière irréversible les générations futures (Faucheux, Hue \& Nicolaï, 2010). La Conférence de Rio de 1992 a établi de manière formelle et officielle une relation négative entre la croissance économique et l'environnement.

Dans le domaine agricole spécifiquement, d'après Khun (1972) cité par (Revéret, Peltier \& Boudier, 1981), la recherche agronomique et la pratique agricole qui en découle, s'accompagnent d'une accumulation effarante d'anomalies: pollution des eaux par les engrais et par les biocides, érosion et compactage des sols, appauvrissement génétique, effets nocifs sur le produit lui-même, épuisement des ressources naturelles non renouvelables etc.

Selon Célestin (2012), le recours d'une part aux variétés nouvelles dites sélectionnées adaptées aux aléas climatiques et, d'autre part à l'usage d'intrants agrochimiques pour accompagner leur production constituent une grave menace pour l'environnement. L'effet de cette agriculture agressive pour l'environnement ne peut donc plus passer sous silence. Ainsi, Pesticide Action Network (2005) rapporte que les pesticides sont utilisés dans les pays en développement en quantités excessives ou inadaptées. Les mesures de protection et d'hygiène lors des traitements phytosanitaires sont souvent négligées par les agriculteurs faute de formations (Kanda et al., 2013; Adechian et al., 2015). Le réceptacle final de toutes les molécules chimiques libérées dans l'environnement est le sol ou les écosystèmes aquatiques (Wang et al., 2009). Elles créent aux populations aquatiques en général et les espèces halieutiques en particulier d'importants dégâts (Agbohessi, Toko \& Kestemont, 2012).

Shelton \& Sears (2001) ont souligné une controverse sur l'impact environnemental des caractères recombinants introduits au bagage génétique des cultures agricoles. Leur étude a révélé la toxicité d'un hybride de maïs transgénique.

Pour Weiskopf \& Lossau (2000), l'agriculture commerciale a réduit aujourd'hui la production autour des variétés hautement performantes. Mais leur diffusion de façon incontrôlée menace la biodiversité (Baco et al., 2007 ; Joshi \& Bauer, 2007 ; Ortman et al., 2001). 
Eu égard à ce développement, l'adoption des variétés améliorées de soja devrait logiquement affecter négativement l'environnement.

\section{Modèle d'impact environnemental}

Afin d'estimer l'effet de l'adoption des variétés améliorées de soja sur l'environnement, le score de durabilité agro-écologique des exploitations agricoles a été régressé sur différentes variables explicatives susceptibles de l'expliquer dont « l'adoption des variétés améliorées de soja », l'objectif étant de voir dans quel sens l'adoption de ces variétés influe-t-elle sur le score. À l'aide d'un adapté du fichier Microsoft Excel inventé par les concepteurs de l'approche de l'Indicateur de Durabilité des Exploitations Agricoles (IDEA) [Vilain et al., 2008], ces scores sont calculés à partir des données d'enquête. Leur calcul est basé sur un système de nombres de points attribués à chaque indicateur avec un plafonnement. Chaque indicateur correspond à certaines pratiques jugées favorables à la durabilité. Il s'agit de scores de valeurs positives et de maximum cent (100) [Vilain, 2008 ; Zahm et al., 2008]. Le modèle estimé se présente comme suit:

$$
\begin{aligned}
\operatorname{Ln}_{\left(\text {duraec }_{i}\right)=} & k_{0}+\rho_{1} \text { alpha }_{i}+\rho_{2} \text { vulgar }_{i}+\rho_{3} \text { sexe }_{i}+\rho_{4} \text { selecvar }_{i} \\
& +\rho_{5} \ln \left(\text { age }_{i}\right)+\rho_{6} \text { accessem }_{i}+\rho_{7} \text { arbori }_{i}+\rho_{8} \text { elevage }_{i} \\
& +\rho_{9} \ln \left(\text { super }_{i}\right)+\rho_{10} \text { sitmat }_{i}+\rho_{11} \text { herbi }_{i}+\rho_{12} \text { fert }_{i} \\
& +\boldsymbol{\rho}_{\mathbf{1 3}} \text { associ }_{\boldsymbol{i}}+\boldsymbol{\rho}_{\mathbf{1 4}} \boldsymbol{P}_{\boldsymbol{i}}+\boldsymbol{\rho}_{\mathbf{1 5}} \boldsymbol{\lambda}_{\boldsymbol{i}}+£
\end{aligned}
$$

avec :

- duraec: indiquant le score de durabilité agro-écologique de l'exploitation agricole ;

- alpha : le statut d'alphabétisation du producteur

- vulgar : le contact avec des agents vulgarisateurs ;

- $\quad$ sexe : le sexe du producteur ;

- selecvar : participation aux expérimentations sur la sélection variétale ;

- $\quad$ age : l'âge du producteur ;

- accessem : l'accessibilité à la semence ;

- $\quad$ arbori : la pratique de l'arboriculture ;

- elevage : la pratique de l'élevage sur l'exploitation ;

- $\quad$ super : la superficie emblavée de soja ;

- $\quad$ sitmat : la situation matrimoniale du producteur.

- herbi : l'utilisation d'herbicide ;

- fert : l'usage d'engrais chimique par le producteur ;

- associ : l'appartenance à une association de producteurs ;

- P : la probabilité d'adoption des variétés améliorées (principale variable explicative) ;

- $\quad \lambda:$ l'inverse du ratio de Mill :

- $\quad \mathrm{k}_{0}$ : la constante ;

- $\quad €$ : le terme d'eurreur. 
Les probabilités prédites d'adoption des variétés améliorées du soja et l'inverse de Mill ont été introduites dans les modèles pour éviter les problèmes d'endogénéité / sélectivité comme l'a préconisé (Heckman, 1979). En effet, la méthode d'estimation de Heckman consiste à d'abord estimer un modèle Probit d'adoption des variétés améliorées (équation de sélection), à récupérer les probabilités prédites d'adoption et $\lambda$ qui est une variable construite à partir des résidus de l'équation de sélection puis à les introduire comme variable explicative dans l'équation d'impact (équation substantielle). Le modèle Probit se présentait comme suit :

$$
\begin{aligned}
& Z_{i}=\alpha_{0}+\alpha_{1} \text { alpha }_{i}+\alpha_{2} \text { vulgar }_{i}+\alpha_{3} \text { sexe }_{i}+\alpha_{4} \text { accessem }_{i}+\alpha_{5} \text { prisem }_{i}+\alpha_{6} \text { risque }_{i} \\
& \quad+\alpha_{7} \text { ssoci }_{i}+\alpha_{8} \text { age }_{i}+\alpha_{9} \text { taille }_{i}+u_{i}
\end{aligned}
$$

Dans cette équation, prisem représente le prix de la semence, risque est la perception du risque, age, l'âge de l'exploitant et taille la taille de son ménage. Le tableau 2 indique les signes attendus des coefficients des variables de l'équation 1.

Dans cette équation, prisem représente le prix de la semence, risque est la perception du risque, age, l'âge de l'exploitant et taille la taille de son ménage. de l'équation 1.

Le tableau 2 indique les signes attendus des coefficients des variables

Tableau 2 : Signes attendus des variables des modèles d'impact environnemental

\section{Variable Définition Signe}

\begin{tabular}{llc}
\hline Ko & Constante & attendu \\
\hline sexe & Sexe du producteur, variable binaire (sexe = 1 si homme, 0 si non) & + +- \\
\hline associ & $\begin{array}{l}\text { appartenance à une association de producteurs, variable binaire (1 si } \\
\text { appartenance, 0 si non) }\end{array}$ & + \\
\hline vulgar & $\begin{array}{l}\text { Contact avec des agents vulgarisateurs, variable binaire (1 si contact, 0 } \\
\text { si non) }\end{array}$ & + \\
\hline accessem & $\begin{array}{l}\text { Disponibilité de la semence, variable binaire (1 si disponible et 0 si } \\
\text { non) }\end{array}$ & + \\
\hline alpha & statut d'alphabétisation, variable binaire (1 si alphabétisé, 0 si non) & + \\
\hline Sitmat & Situation matrimoniale du producteur (1 si pas de progéniture, 0 si non) & - \\
\hline fert & $\begin{array}{l}\text { Utilisation d'engrais chimiques, variable binaire (1 si utilisation et 0 si } \\
\text { non) }\end{array}$ & - \\
\hline herbi & Utilisation d'herbicide, variable binaire (1 si utilisation et 0 si non) & - \\
\hline arbori & Pratique d'arboriculture (1 si pratique, 0 si non) & + \\
\hline Elevage & Pratique de l'élevage (1 si pratique, 0 si non) & + \\
\hline Age & Age du producteur, variable quantitative & + \\
\hline $\mathbf{P}$ & probabilité prédite d'adoption des variétés améliorées & + \\
\hline \multicolumn{1}{c}{$\boldsymbol{\lambda}$} & l'inverse de Mill & \\
\hline
\end{tabular}

Source : réalisé par les auteurs (Mars 2018) 


\section{Indicateurs pris en compte dans le calcul du score de durabilité agro- écologique}

La durabilité agro-écologique analyse la capacité de l'exploitation à concilier valorisation efficace du milieu et minimisation du coût écologique (Zucchini et al., 2016). Elle est fondée sur des indicateurs qui se répartissent en trois composantes à savoir : la diversité domestique, l'organisation spatiale et les pratiques agricoles. Le principe est qu'il est possible de quantifier chaque composante puis d'agréger les informations pour obtenir un score de durabilité de l'exploitation. La pertinence des différents indicateurs est donnée par Cabot et al. (2018).

\section{La diversité domestique}

La diversité domestique rend compte de la capacité de l'exploitation à préserver la fertilité du sol et l'écosystème (Zucchini et al., 2016). Cette composante regroupe la diversité des cultures annuelles, la diversité des cultures pérennes, la diversité animale puis la valorisation et la conservation du patrimoine génétique.

$\checkmark$ Al-Diversité des cultures annuelles

Objectif : Cet indicateur vise la préservation de la biodiversité végétale car s'il est diversifié, le système sera capable de rompre les cycles parasitaires, de réduire la sensibilité du système aux aléas climatiques et de protéger les sols contre l'érosion.

\section{$\checkmark$ A2-Diversité des cultures pérennes}

Objectif : Elle vise la préservation d'espèces pérennes qui jouent un rôle important dans l'équilibre de l'agroécosystème. En effet, ces espèces contribuent au maintien de la fertilité des sols, à leur protection contre l'érosion, l'amélioration de la qualité des eaux de surface par une action de filtration biologique. Ces fonctions stabilisatrices sont cependant effectives au-delà d'un seuil de 10\% de la surface agricole utile (SAU).

\section{$\checkmark$ A3-Diversité animale}

Objectif : Il s'agit d'un indicateur qui priorise la présence d'élevage au sein de l'exploitation agricole. Il permet d'utiliser les déjections animales pour entretenir la fertilité des sols à long terme et de réduire l'intégration d'intrants exogènes.

$\checkmark$ A4-Valorisation et conservation du patrimoine génétique

Objectif : L'indicateur vise la préservation de la biodiversité domestique de la région (par la protection des races et des variétés menacées de disparition) [Ahouangninou, 2013]. Selon, Cabot et al. (2018), il faut éviter l'érosion génétique locale au profit d'une standardisation mondiale car cela peut compromettre la capacité d'adaptation de la ressource agricole aux conditions locales. 


\section{L'organisation spatiale}

L'organisation de l'espace apprécie si l'exploitation est susceptible de gérer et de valoriser le périmètre (Zucchini et al., 2016). Elle prend en compte les composantes : assolement, dimension des parcelles, gestion des matières organiques et zones de régulation écologique.

\section{$\checkmark$ A5-Assolement}

Objectif : Cet indicateur poursuit l'objectif d'une utilisation optimale de l'espace afin d'assurer une complémentarité des espèces cultivées.

$\checkmark$ A6-Dimension des parcelles

Objectif: L'indicateur se préoccupe de la cohérence technique de l'exploitation et de la transmissibilité et l'efficience des ressources engagées.

$\checkmark$ A7-Gestion des matières organiques

Objectif : Il s'attache à la préservation de la biodiversité ainsi qu'à la bonne qualité des sols et celle de la vie.

\section{$\checkmark$ A8-Zone de régulation écologique}

Objectif : L'objet de cet indicateur est de valoriser le maintien et/ou la mise en place sur l'exploitation, de zones naturelles dont les seuls objectifs sont d'ordre écologique (filtration des eaux, prolifération d'une biodiversité...).

\section{Les pratiques agricoles}

Quant à la composante " pratiques agricoles », elle détermine l'impact environnemental des pratiques culturales auxquelles a recours l'exploitation. Les indicateurs qui la composent sont : la fertilisation, la gestion des effluents organiques liquides, l'utilisation des pesticides, les traitements vétérinaires, la protection de la ressource sol, la gestion de la ressource en eau et dépendance énergétique de l'exploitation (Zucchini et al., 2016).

\section{$\checkmark$ A12-Fertilisation}

Objectif : Il s'agit pour l'exploitation, de produire sans polluer (préserver la biodiversité des sols et la qualité de l'eau) ni gaspiller. En effet, la fertilisation minérale détruit la structure du sol et augmente les risques d'érosion et de pollution des ressources en eau. Par contre, lorsque la fertilisation est organique, elle permet une bonne activité biologique du sol.

\section{$\checkmark$ A13-Effluent organique liquide}

Objectif : L'exploitation doit pouvoir bien gérer les productions d'effluents qui engendrent des risques importants de pollution du sol et surtout des eaux.

\section{$\checkmark$ A14-Pesticide}

Objectif : Cet indicateur propose d'évaluer les pratiques du producteur sur l'utilisation des pesticides pour protéger les végétaux. En effet, lorsqu'ils sont fortement utilisés, les pesticides infectent le sol et les eaux avec pour conséquences des préjudices à l'environnement (ruine des insectes pollinisateurs, intoxication alimentaire etc.). 


\section{$\checkmark$ A15-Traitement vétérinaire}

Objectif : Il s'agit de promouvoir des pratiques assurant un bien-être aux animaux et une qualité de la production.

$\checkmark$ A16-Protection de la ressource sol

Objectif : L'objectif de cet indicateur est d'encourager les mesures visant à protéger les sols contre l'érosion (dispositifs anti-érosifs, présence de couverture végétale permanente ou quasi permanente, travail du sol sans labour etc.).

$\checkmark$ A17-Gestion de la ressource en eau

Objectif : L'indicateur «Gestion de la ressource en eau » vise à limiter le gaspillage de la ressource eau. En effet, la pratique d'irrigation constitue un prélèvement net sur la ressource et ne permet pas une restitution. De plus, l'irrigation augmente le taux de minéralisation de la matière organique des sols, ce qui entraîne de fertilité avec le temps.

$\checkmark$ A18-Dépendance énergétique

Objectif : Il vise à évaluer la dépendance énergétique des exploitations agricoles afin de promouvoir à terme l'autonomie énergétique (production des énergies renouvelables). Ceci conduira inévitablement à une économie d'énergie.

\section{Résultats}

\section{Statut d'adoption et valeur du score de durabilité agro-écologique}

Le score de durabilité agro-écologique affiche une valeur moyenne de 57,03 (avec un maximum de 76 et un minimum de 37) sur 100 et de 54,88 (avec un maximum de 73 et un minimum de 36) sur 100 respectivement pour les adoptants et les non adoptants de variétés améliorées de soja (tableau 3). Alors, qu'elles soient adoptantes ou non, les exploitations sont très peu durables écologiquement en moyenne. En effet, les scores obtenus valent à peine (en moyenne) la moyenne théorique (50) du score de durabilité agroécologique. Le test de comparaison de moyennes de Student ( $\mathrm{p}$-value $>0,05$ ) appliqué aux scores confirme la similarité des deux groupes (adoptants et non adoptants) au point de vue durabilité agro-écologique (tableau 4).

Tableau 3: Valeurs moyennes des indicateurs

\begin{tabular}{|c|c|c|c|c|}
\hline \multirow[b]{2}{*}{ Composantes } & \multirow[b]{2}{*}{ Indicateurs } & \multicolumn{2}{|c|}{ Moyennes } & \multirow[b]{2}{*}{$\begin{array}{c}\text { Maximum } \\
\text { possible }\end{array}$} \\
\hline & & Adoptants & $\begin{array}{c}\text { Non } \\
\text { adoptants }\end{array}$ & \\
\hline \multirow{5}{*}{$\begin{array}{c}\text { Diversité } \\
\text { domestique }\end{array}$} & Diversité des cultures annuelles & 12,75 & 12 & 14 \\
\hline & Diversité des cultures pérennes & 4,13 & 2,85 & 14 \\
\hline & Diversité animale & 1,02 & 0,71 & 14 \\
\hline & $\begin{array}{l}\text { Valorisation et conservation du } \\
\text { patrimoine génétique }\end{array}$ & 2,38 & 3,25 & 04 \\
\hline & Composante & 20,21 & 18,82 & 33 \\
\hline \multirow{2}{*}{$\begin{array}{l}\text { Organisation } \\
\text { spatiale }\end{array}$} & Assolement & 5,76 & 5,35 & 08 \\
\hline & Dimension des parcelles & 6 & 5,95 & 06 \\
\hline
\end{tabular}




\begin{tabular}{ccccc}
\hline & $\begin{array}{c}\text { Gestion des matières } \\
\text { organiques }\end{array}$ & 0 & 0 & 05 \\
& Zone de régulation écologique & 1 & 0.85 & 12 \\
\cline { 2 - 5 } Composante & $\mathbf{1 2 , 7 6}$ & $\mathbf{1 2 , 1 6}$ & $\mathbf{3 3}$ \\
\cline { 2 - 5 } & Fertilisation & 6,72 & 6,76 & 8 \\
\cline { 2 - 5 } Pratiques & Effluent organique liquide & 4 & 4 & 04 \\
\cline { 2 - 5 } agricoles & Pesticide & 0,75 & 0,66 & 13 \\
\cline { 2 - 5 } & Traitement vétérinaire & 0,13 & 0,05 & 03 \\
\cline { 2 - 5 } & Protection de la ressource sol & 0,44 & 0,41 & 05 \\
\cline { 2 - 5 } & Gestion de la ressource en eau & 4 & 4 & 04 \\
\cline { 2 - 5 } & Dépendance énergétique & 8 & 8 & 10 \\
\cline { 2 - 5 } Score de durabilité agro-écologique & $\mathbf{2 4 , 0 5}$ & $\mathbf{2 3 , 9 0}$ & $\mathbf{3 4}$ \\
\hline Composante & $\mathbf{5 4 , 8 8}$ & $\mathbf{1 0 0}$ \\
\hline
\end{tabular}

Source : Réalisé par les auteurs à partir des données d'enquête (Mars 2018)

Figure 1: Valeurs du score de durabilité agro-écologique suivant le statut d'adoption

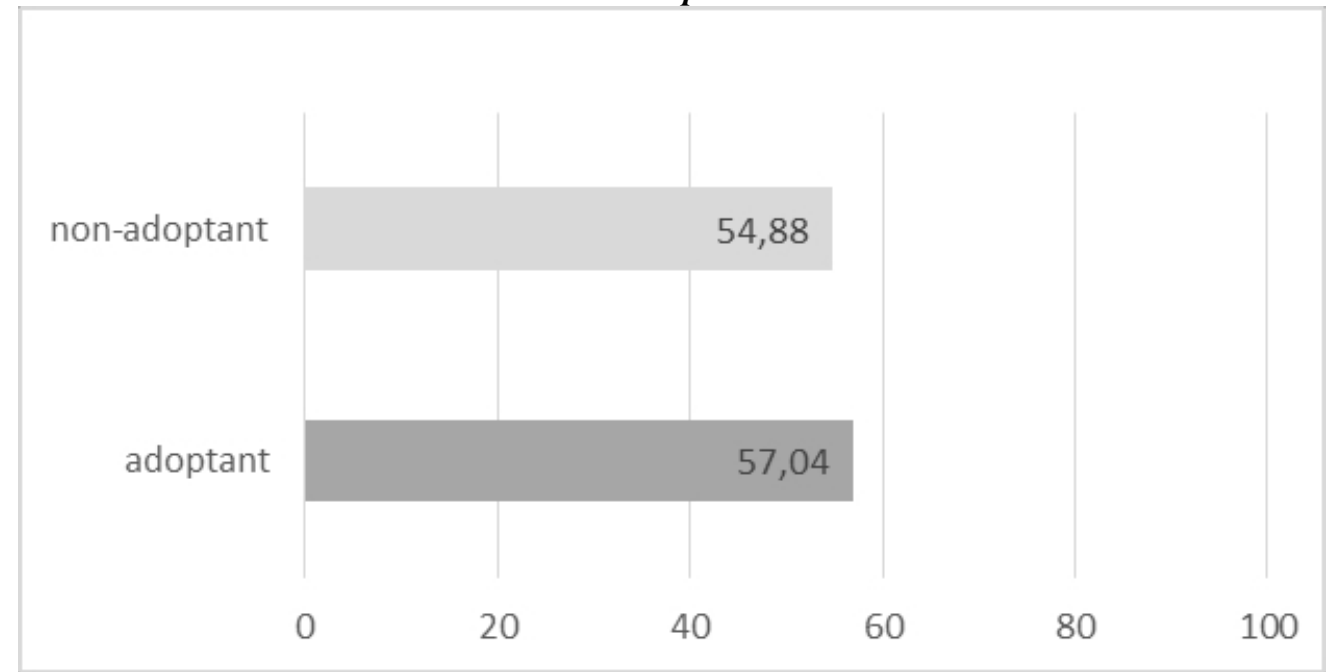

Source : Réalisée par les auteurs à partir des données d'enquête (Mars 2018)

Une analyse par composante permet de tirer la même conclusion. En effet, on constate que les exploitations ayant adopté les variétés améliorées de soja n'ont pas une production moins diversifiée que celles ne les ayant pas adopté (tableau 3). Les valeurs obtenues par les deux groupes sont respectivement 20,21 et 18,82 valant $61,24 \%$ et $57 \%$ du maximum possible pour la composante de diversité agro-écologique. Ces valeurs de la composante sont loin de garantir la préservation et la fertilité du sol et l'écosystème. En dehors de l'objectif de préservation de la biodiversité qui est atteint $(91,07 \%$ et $85,71 \%$ du maximum possible pour la diversité des cultures annuelles respectivement chez les adoptants et non adoptants), les exploitations ne bénéficient ni des fonctions stabilisatrices que pourraient 
apporter les cultures pérennes ni de l'évite de l'érosion génétique locale sauf celles des non adoptants $(81,25 \%$ du maximum possible pour l'indicateur «Valorisation et conservation du patrimoine génétique »).

Figure 2: Valeurs des indicateurs de la composante «diversité domestique » et maximum possible

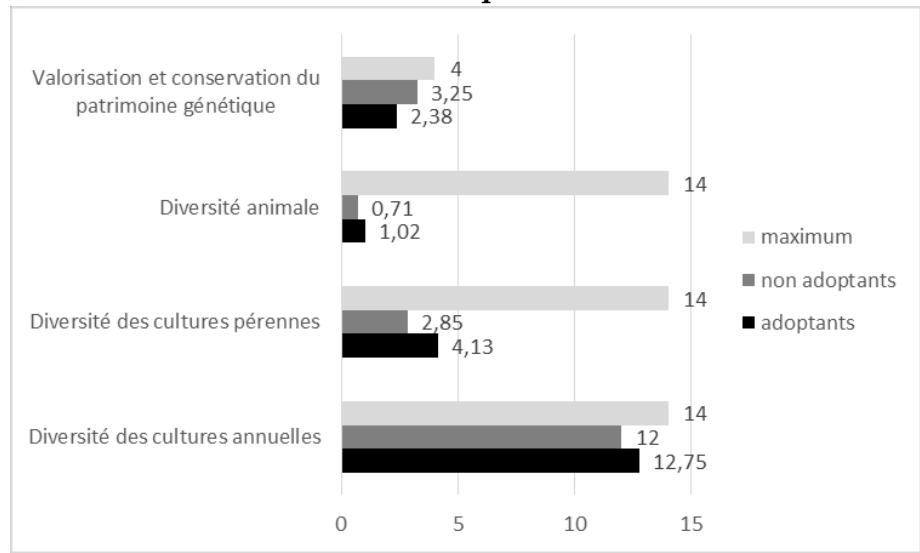

Source : Réalisée par les auteurs à partir des données d'enquête (Mars 2018)

Considérant la composante " organisation spatiale », les valeurs de 12,76 et 12,16 soit $38,66 \%$ et $36,84 \%$ (tableau 3 ) du maximum possible respectivement chez les adoptants et les non adoptants ne démontrent pas une bonne gestion et une bonne valorisation du périmètre au niveau des deux groupes. La faiblesse de cette composante sur les exploitations est déterminée par l'absence aussi bien de la gestion des matières organiques que de zones de régulation écologiques. Par contre, on assiste à une utilisation optimale de l'espace et à une cohérence technique de l'exploitation ainsi qu'à l'efficience des ressources engagées.

Figure 2: Valeurs des indicateurs de la composante « organisation spatiale » et maximum possible

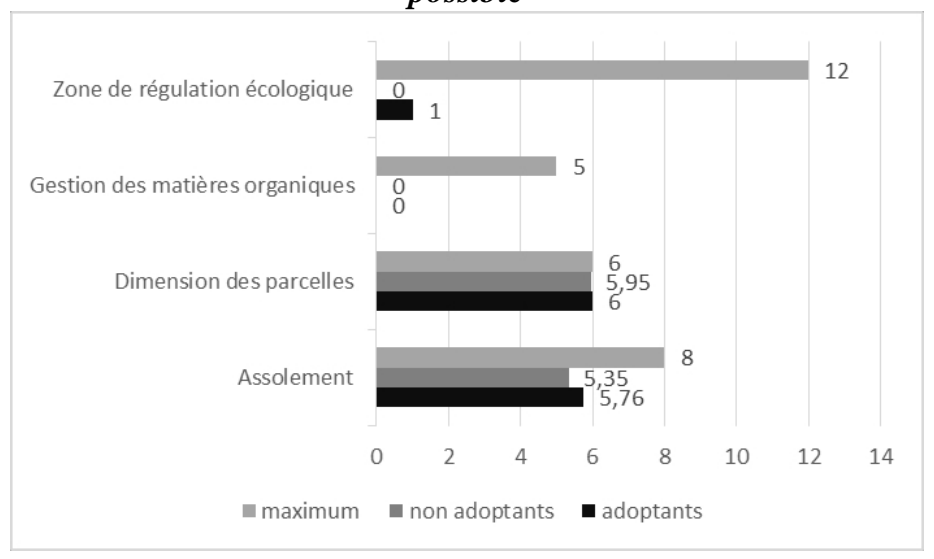

Source : Réalisée par les auteurs à partir des données d'enquête (Mars 2018) 
Concernant enfin la composante "pratiques agricoles », les valeurs obtenues sont de 24,05 et de 23,90 (tableau 3) soit 70,73\% et 70,29\% du maximum possible respectivement pour les adoptants et les non adoptants. Ces niveaux élevés de la composante s'expliquent par l'absence d'utilisation de fertilisants chimiques et de libération d'effluents organiques liquides d'une part, puis par une bonne gestion de la ressource en eau et la non dépendance énergétique des exploitations d'autre part. Ainsi, les pratiques culturales des exploitants limitent plus ou moins la pollution de l'environnement.

Figure 3: Valeurs des indicateurs de la composante «pratique agricole » et maximum

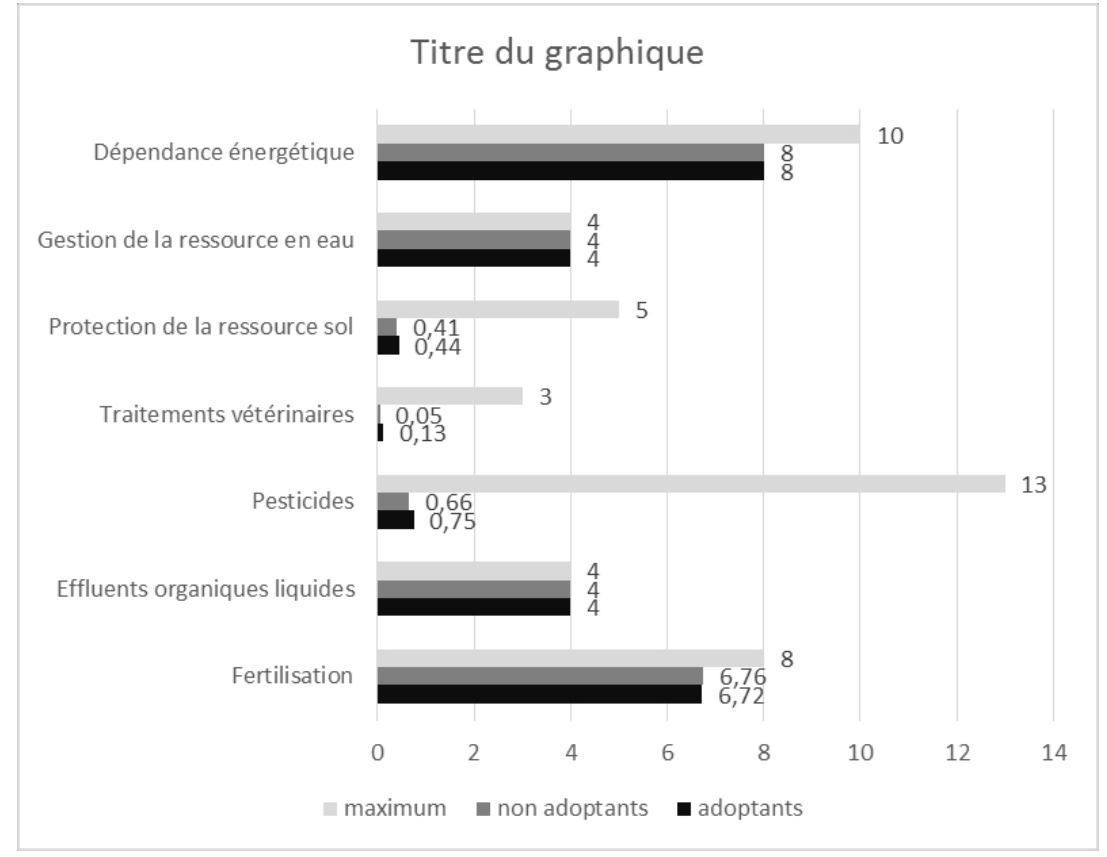

Source : Réalisée par les auteurs à partir des données d'enquête (Mars 2018)

Tableau 4: Résultats du test de comparaison de moyennes de Student

\begin{tabular}{lcc}
\hline Adoptions & Observations & Moyennes \\
\hline Non adoptants & 117 & 54.88889 \\
\hline Adoptants & 102 & 57.03922 \\
\hline p-value & \multicolumn{2}{c}{0.0526}
\end{tabular}

Source : Réalisé par les auteurs à partir des données d'enquête (Mars 2018)

Impact de l'adoption des variétés améliorées de soja sur le score de durabilité agro-écologique

L'estimation du modèle d'impact environnemental (avec l'estimateur du maximum de vraisemblance) affiche un coefficient non significatif pour l'inverse du rapport de mill (Prob LR test $[$ rho $=0]>0,05$ ), ce qui signifie que l'adoption des variétés améliorées de soja n'est pas endogène dans le modèle 
(tableau 5). Un modèle de régression linéaire multiple permet, dans ces conditions, d'obtenir des estimateurs non biaisés. Les résultats des estimations sont présentés dans le tableau 5. La probabilité de Fischer (0.0000) étant inférieure à 5\%, on n'a aucune raison de rejeter l'hypothèse alternative selon laquelle tous les coefficients ne sont pas tous égaux à zéro. Les variables indépendantes expliquent à $48 \%$ les variations du score de durabilité agroécologique. Cette variable est en effet positivement expliquée par le sexe, la pratique de l'arboriculture et celle de l'élevage. Cela signifie que le fait d'être de sexe masculin, de pratiquer l'arboriculture ou l'élevage améliore le score de durabilité agro-écologique. En d'autres termes, chacune de ces variables améliore la qualité de l'environnement sur l'exploitation agricole.

Par contre, les résultats de la présente étude montrent que la pratique de la fertilisation chimique réduit le score de durabilité agro-écologique c'est-à-dire qu'elle pollue l'environnement.

Par ailleurs, l'adoption des variétés améliorées de soja n'affecte pas la qualité de l'environnement dans le département du Borgou car le coefficient de cette variable n'est pas significatif dans le modèle d'impact.

Tableau 5. Résultats de l'estimation du modèle d'impact environnemental de l'adoption des variétés améliorées de soja

\begin{tabular}{|c|c|c|c|c|}
\hline \multirow{2}{*}{ Variables } & \multicolumn{2}{|c|}{ Heckman } & \multicolumn{2}{|c|}{ Regress } \\
\hline & coef. & $\mathrm{P}>\mathrm{Z}$ & coef. & $\mathrm{P}>\mathrm{Z}$ \\
\hline \multicolumn{5}{|l|}{ Lscore } \\
\hline Alpha & -0.017 & 0.503 & 0.006 & 0.719 \\
\hline Vulgar & -0.005 & 0.840 & -0.005 & 0.814 \\
\hline Sexe & 0.101 & $0.028 * *$ & 0.127 & $0.000 * * *$ \\
\hline Selecvar & 0.047 & 0.385 & 0.011 & 0.793 \\
\hline Lage & -0.072 & 0.148 & 0.028 & 0.351 \\
\hline Accessem & -0.080 & $0.062 *$ & $-0.066^{*}$ & $0.051 *$ \\
\hline Arbori & 0.117 & $0.000 * * *$ & 0.112 & $0.000 * * *$ \\
\hline Elevage & 0.136 & $0.000 * * *$ & 0.094 & $0.000 * * *$ \\
\hline Lsuper & -0.006 & 0.660 & 0.015 & 0.184 \\
\hline Sitmat & -0.057 & 0.487 & -0.019 & 0.485 \\
\hline Herbi & -0.020 & 0.437 & -0.037 & $0.054^{*}$ \\
\hline Fert & -0.166 & $0.004 * * *$ & -0.133 & $0.002 * * *$ \\
\hline Associ & -0.025 & 0.372 & 0.0261 & 0.164 \\
\hline Sem & & & 0.033 & $0.059 *$ \\
\hline Cons & 4.328 & $0.000 * * *$ & 3.759 & $0.000 * * *$ \\
\hline \multicolumn{5}{|l|}{ Sem } \\
\hline Alpha & 0.150 & 0.481 & & \\
\hline Vulgar & -0.071 & 0.786 & & \\
\hline Sexe & 0.818 & $0.014 * *$ & & \\
\hline Accessem & 1.144 & $0.000 * * *$ & & \\
\hline Prisem & 0.004 & $0.000 * * *$ & & \\
\hline Risque & -0.852 & $0.007 * * *$ & & \\
\hline
\end{tabular}




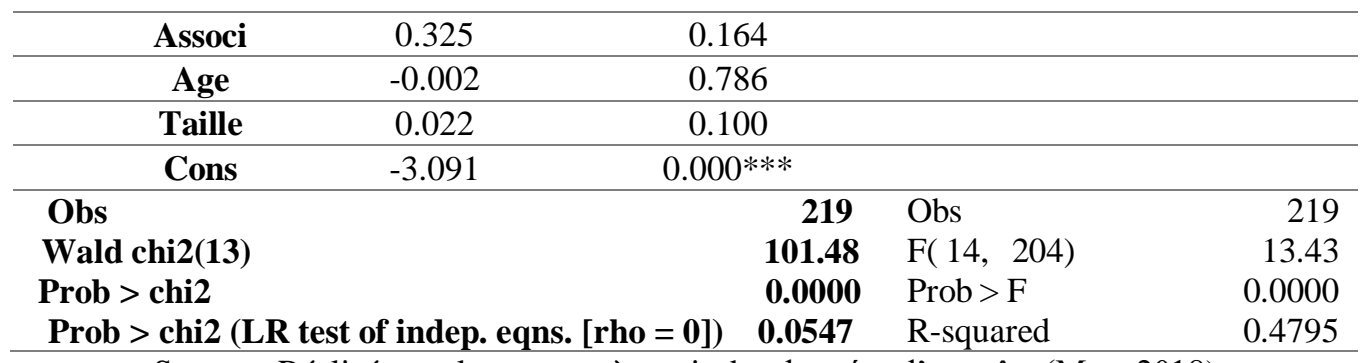

Source : Réalisée par les auteurs à partir des données d'enquête (Mars 2018)

\section{Discussion}

Les variables explicatives (sexe, pratique de l'arboriculture et pratique d'élevage) expliquent les variations du score de durabilité agro-écologique. Ces résultats confirment ceux de Cabot et al. (2018) pour qui les espèces pérennes contribuent au maintien de la fertilité des sols, à leur protection contre l'érosion, à l'amélioration de la qualité des eaux de surface par une action de filtration biologique tandis que l'élevage permet d'utiliser les déjections animales pour entretenir la fertilité des sols à long terme et de réduire l'intégration d'intrants exogènes.

Par contre, les résultats de cette étude montrent que la pratique de la fertilisation chimique réduit le score de durabilité agro-écologique c'est-à-dire qu'elle pollue l'environnement. C'est ainsi que Cabot et al. (2018) affirment que la fertilisation minérale détruit la structure du sol et augmente les risques d'érosion et de pollution des ressources en eau. L'utilisation de l'herbicide, bien entendu largement répandue dans la zone d'étude ne détériore pas le score de durabilité agro-écologique. Bien que surprenant, cet état de choses s'explique par le fait que les producteurs respectent bien le dosage recommandé.

Par ailleurs, l'adoption des variétés améliorées de soja n'affecte pas la qualité de l'environnement dans le département du Borgou. Un tel résultat est tout de même surprenant car théoriquement, on s'attend à ce que l'utilisation de nouvelles techniques génétiques en agriculture ait des répercussions sur l'environnement. Cela s'explique en effet par le fait que le soja amélioré n'est pas accompagné de son paquet technologique à savoir le Chlorure de potassium $(\mathrm{KCl})$ et le triple superphosphate (TSP) dans la zone d'étude. Ces engrais spécifiques sont quasi inexistants et les producteurs préfèrent produire sans fertilisants plutôt que d'utiliser d'autres types d'engrais (pratique proche de l'agriculture Biologique). Ce résultat contraste avec ceux de Célestin (2012) qui a montré que l'adoption des variétés améliorées pollue l'environnement ou ceux de Joshi \& Bauer (2007) de Ortman et al. (2001); et de Bardsley \& Thomas (2005) qui ont quant à eux noté une perte de biodiversité. Mais il rejoint ceux de Steele et al. (2009) qui ont justifié l'absence d'incompatibilité entre le développement des variétés modernes et 
la préservation des espèces anciennes ou encore ceux de Fu (2006) pour qui l'adoption de nouvelles variétés de riz a favorisé l'augmentation de la diversité génétique globale.

\section{Conclusion}

Il était question, dans ce papier, de vérifier l'hypothèse selon laquelle la production des variétés améliorées de soja affecte négativement l'environnement dans le département du Borgou au Nord du Bénin. Pour ce faire, les scores de durabilité agro-écologique des exploitations agricoles ont été calculés puis régressés sur les facteurs susceptibles de les expliquer dont l'adoption de ces variétés. Au terme des analyses, il convient de retenir que l'adoption des variétés améliorées de soja n'affecte pas l'environnement dans le département du Borgou. Les divers acteurs du développement des filières agricoles peuvent alors promouvoir ces variétés à travers les institutions de recherche et les organisations professionnelles d'agriculteurs. Pour cela, il faudra développer la production des semences améliorées de soja et sensibiliser les producteurs sur l'intérêt d'adopter ces technologies.

$\mathrm{Si}$ cette étude a pu montrer que la production des nouvelles technologies de soja ne pollue pas l'environnement dans la zone d'étude, elle n'a toutefois pas pris en comptes la diversité des exploitations agricoles. Des recherches ultérieures pourraient introduire la caractérisation des exploitations agricoles à base de soja amélioré.

\section{References:}

1. Adechian S., Nasser Baco M., Akponikpe I., Imorou Toko I., Egah J. \& Affoukou K. (2015). Les pratiques paysannes de gestion des pesticides sur le maïs et le coton dans le bassin cotonnier du Bénin. [VertigO] La revue électronique en sciences de l'environnement, 15(2).

2. Agbohessi T.P., Toko I.I. \& Kestemont P. (2012). État des lieux de la contamination des écosystèmes aquatiques par les pesticides organochlorés dans le Bassin cotonnier béninois. Cahiers Agricultures, 21(1), 46-56.

3. Ahouangninou C. (2013). Durabilité de la production maraîchère au Sud-Bénin : un essai de l'approche (PhD Thesis). UAC, 333 p.

4. Akomagni L.A. \& Ichola J. (2017). Étude diagnostique du fonctionnement du marché de l'anacarde et perspectives sur les politiques nationales de développement de la filière au Bénin, $100 \mathrm{p}$.

5. Baco M.N., Biaou G., Pinton F. \& Lescure J.P. (2007). Les savoirs paysans traditionnels conservent-ils encore l'agrobiodiversité au Bénin? Biotechnologie, Agronomie, Société et Environnement, 11(3), 201-210. 
6. Bardsley D. \& Thomas I. (2005). In situ agrobiodiversity conservation for regional development in Nepal. Geojournal, 62(1-2), 27-39.

7. Batamoussi M.H., Boulga J., Yolou I., Tokore J.S.B.O.M., Lafia K. \& Issa A. (2016). Analysis of peasant practices for soy production (Glycine max) in the district of Kalale (Northern-Bénin) : Implication for their improvement. International Journal of Innovation and Applied Studies, 25(2), 501-509.

8. Cabot V., Danflous J.-P., Le Bellec F., Lesage S., Thomas P., \& Zahm F. (2018). La méthode IDEA Réunion-IDEA RUN-Indicateurs de durabilité des exploitations agricoles, $154 \mathrm{p}$.

9. Célestin A.Y. (2012). Production agricole et changement climatique : vers une tragédie des comportements paysans à Tiassalé? European Scientific Journal, ESJ, 8(16). Consulté à l'adresse http://eujournal.org/index.php/esj/article/view/258

10. Chogou S.K., Okry F., Santos F. \& Hounhouigan, D. J. (2018). Efficacité technique des producteurs de soja du Bénin. Annales des Sciences Agronomiques, 22(1), 93-110.

11. Faucheux S., Hue C. \& Nicolaï I. (2010). TIC et développement durable : Les conditions du succès. De Boeck Supérieur, Université de Bruxelles, $222 \mathrm{p}$.

12. Fu Y.-B. (2006). Impact of plant breeding on genetic diversity of agricultural crops: Searching for molecular evidence. Plant Genetic Resources, 4(1), 71-78.

13. Heckman J.J. (1979). Sample Selection Bias as a Specification Error. Econometrica, 47(1), 153-161.

14. Joshi G. R. \& Bauer S. (2007). Cultivation and the loss of rice landraces in the Terai region of Nepal. Plant Genetic Resources, 5(1), $1-6$.

15. Kanda M., Djaneye-Boundjou G., Wala K., Gnandi K., Batawila K., Sanni A. \& Akpagana K. (2013). Application des pesticides en agriculture maraichère au Togo. VertigO-la revue électronique en sciences de l'environnement, 13(1).

16. Konnon D.D. \& ahoueya J. (2017). État des lieux sur la filière soja au Bénin et identification de ses chaînes de valeurs ajoutées (CVA) porteuses, RAPPORT PROVISOIRE. MAEP-Bénin, 133 p.

17. Kuhn T. (1972). La structure des révolutions scientifiques, 26 p.

18. Njonjo Assani J. (2014). Contribution des nouvelles technologies de soja au bien-etre des ménages agricoles - Recherche Google. Consulté 7 juin 2018, à l'adresse https://www.google.bj/

19. Ortman E.E., Barry B.D., Buschman L.L., Calvin D.D., Carpenter J., Dively G.P., Higgin S.R.A. (2001). Transgenic insecticidal corn : The 
agronomic and ecological rationale for its use. BioScience, 51(11), 900-903.

20. Pesticide Action Network. (2005). Étude d'impact socio-économique, sanitaire et environnemental de l'utilisation des POPs à Davié au Nord de Lomé (région Maritime) (p. 37). Lomé: IPEP, PAN Togo.

21. Revéret J.-P., Peltier J. \& Boudier H. (1981). De l'agriculture conventionelle à l'agriculture écologique, vers un nouveau paradigme. Sociologie et sociétés, 13(1), 49-62.

22. Shelton A.M. \& Sears M.K. (2001). The monarch butterfly controversy: Scientific interpretations of a phenomenon. The Plant Journal, 27(6), 483-488.

23. Steele K.A., Gyawali S., Joshi K.D., Shrestha P., Sthapit B.R., \& Witcombe J.R. (2009). Has the introduction of modern rice varieties changed rice genetic diversity in a high-altitude region of Nepal? Field Crops Research, 113(1), 24-30.

24. Thibault L. (1970). Adam Smith, Recherche sur la nature et les causes de la richesse des nations. Dissertation, Publications Pimido, 2 p.

25. Vilain L., Boisset K., Girardin P., Guillaumin, A., Mouchet, C., Viaux, P., \& Zahm, F. (2008). La méthode IDEA : indicateurs de durabilité des exploitations agricoles: Guide d'utilisation. Consulté 10 septembre 2019, à l'adresse https://prodinra.inra.fr/?locale=fr\#!ConsultNotice: 33898

26. Vilain L. (2008). La méthode IDEA : Indicateurs de durabilité des exploitations agricoles. Educagri éditions, $184 \mathrm{p}$.

27. Waaub J.-P. (1991). Croissance économique et développement durable: vers un nouveau paradigme du développement. Environnement et développement : questions éthiques et problèmes socio-politiques, $374 \mathrm{p}$.

28. Wang C., Lu G., Cui J., \& Wang P. (2009). Sublethal effects of pesticide mixtures on selected biomarkers of Carassius auratus. Environmental toxicology and pharmacology, 28(3), 414-419.

29. Weiskopf B., \& Lossau A. (2000). Gestion de l'agro-biodiversité dans les zones rurales. GTZ, 12 p.

30. Zahm F., Viaux P., Vilain L., Girardin P. \& Mouchet, C. (2008). Assessing farm sustainability with the IDEA method-from the concept of agriculture sustainability to case studies on farms. Sustainable development, 16(4), 271-281.

31. Zucchini E., Faye A., Ngom Y. \& Diémé R. (2016). METHODE IDEA, 39 p. 